\title{
Infertility influencers: an analysis of information and influence in the fertility webspace
}

\author{
Jennifer K. Blakemore ${ }^{1}$ - Arielle H. Bayer ${ }^{2}$. Meghan B. Smith ${ }^{3}$ - James A. Grifo ${ }^{1}$
}

Received: 15 February 2020 / Accepted: 24 April 2020 / Published online: 7 May 2020

(C) Springer Science+Business Media, LLC, part of Springer Nature 2020

\begin{abstract}
Purpose To examine fertility-related social media accounts and influencers on two social media platforms.

Methods The search function of Twitter (TW) and Instagram (IG) was used to generate a list of accounts with the terms: fertility, infertility, ttc, egg freezing, ivf, endometriosis, and reproductive. Accounts not in English, in private, with no posts in $>1$ year, or with content unrelated to search terms were excluded. Accounts were assessed for author type; REI board certification (REI-BC); influencer (INF) status (> $10 \mathrm{~K}$ followers on IG; verified check mark on TW); account demographics; and content in last 5 posts. Statistical analysis included unpaired $t$ tests, a classification and regression tree (CART) analysis, and stepwise multiple logistic regression.

Results Seven hundred ten accounts were identified and 537 (278 TW, 259 IG) were included. Account types included societies, clinics, physicians, patients, groups, and "other." Instagram content (1290 posts reviewed) was primarily personal stories (31.7\%) or inspiration/support (23.7\%). Twitter content (1390 posts reviewed) was mostly promotion (28.2\%) and research/education $(20.2 \%)$. Thirty-nine accounts (12.5\%) were influencers. Fertility influencers were most often awareness/support accounts (59.8\% TW, 25.0\% IG), patients (12.8\% TW, 25\% IG), or other (17.9\% TW, 21.0\% IG). Only 7.7\% TW and 7.1\% IG INFs were board-certified REI physicians. The best predictor for classification as an influencer was high activity ( $>50$ posts/month TW, > 10 posts/month IG).

Conclusion As patients increasingly utilize social media to obtain and engage with health information, it is critical to understand the fertility-related SM landscape. This understanding may help to successfully enhance relationships with patients and ensure dissemination of accurate information.
\end{abstract}

Keywords Infertility $\cdot$ Social media $\cdot$ Influencer $\cdot$ Patient education

Research of Arielle H. Bayer was performed while at New York University and manuscript completed while at Albert Einstein College of Medicine

Jennifer K. Blakemore

Jennifer.Blakemore@nyulangone.org

1 New York University Langone Fertility Center, 660 First Avenue, Fifth Floor, New York, NY 10016-3295, USA

2 Division of Reproductive Endocrinology \& Infertility, Albert Einstein College of Medicine/Montefiore Medical Center, Bronx, NY 10461, USA

3 Division of Reproductive Endocrinology and Infertility, University of Southern California, Los Angeles, CA 90033, USA

\section{Introduction}

Social media has transformed communication and cultural influence, through the use of electronic communication to share information, ideas, personal messages, and other content on platforms such as Twitter and Instagram. Healthcare is not immune to this cultural shift. Armed with smartphones, up to $80 \%$ of Americans have searched the internet for health-related information and up to $40 \%$ doubt a professional opinion when it conflicted with web-based findings [1, 2]. However, little is known about the source and substance of medical information on social media platforms. Even less is known about which sources are the most influential. As a response to the proliferation of these unsourced (and potentially unreliable) accounts, a growing number of physicians and professional societies have turned to social media to disseminate evidence-based information and to promote their practices. 
Reproductive-aged women are avid consumers of social media and the internet, with $73 \%$ of adult women in the USA using social media [3]. Patients generally view the social media healthcare space favorably - a survey of patients in an infertility clinic showed $79.9 \%$ of respondents felt social media benefitted the patient experience [4]. A recent study of couples desiring children and actively trying to conceive found that participants were most likely to seek fertilityrelated information on the internet as a first source [5]. Yet, these same participants overestimated a woman's reproductive lifespan and demonstrated little knowledge regarding the correct "fertile window" within the menstrual cycle [5]. Thus, despite access to "information," a knowledge gap may remain.

One cultural phenomenon that has risen from the proliferation of social media is the role of the "social media influencer." An "influencer" is a person or brand who has a significant digital footprint in their online community, as defined by platform, and whose opinions drive thoughts and trends within that community. Indeed, an analysis of Twitter users found that influencers rival their own supports (e.g., friends) in building user trust, with $56 \%$ of users trusting the opinion of an influencer over friends [6]. While physicians and healthcare organizations would be the most ideal influencers within the healthcare social media space, building influence is intricate and time consuming. Busy physicians often do not have the time to cultivate consumer engagement, create content, network, and perform frequent maintenance.

Given the ubiquity of social media, physician influencers could be a powerful tool to provide accurate and useful medical information within social media. However, there is a need to better understand the topography of social media medical information and sources, as well as factors that help determine which sources are the most influential. Therefore, we sought to examine (i) individual social media accounts related to fertility/infertility on two platforms-Instagram and Twitter - and (ii) the factors associated with the accounts that attain influencer status as defined by social media platform.

\section{Materials and methods}

\section{Design}

We conducted a cross-sectional analysis of public, fertilityrelated, social media accounts on Twitter (TW) and Instagram (IG) between March 31, 2019, and April 7, 2019. We chose a "typical" week in the social media world instead of a time where fertility social media might be more active or have more outreach, such as around the annual American Society of Reproductive Medicine (ASRM) meeting or National Infertility Awareness Week (NIAW). While other platforms, such as Facebook, are more popular overall, Instagram and Twitter were chosen for their consistent use by women of reproductive age [7]. Furthermore, since accounts and posts are likely influenced by news and events, the timeframe of 1 week was chosen to ensure all variables were collected within the same temporal window. The NYU Langone Health self-certification form from the Institutional Review Board was used to determine that the research question and design for this study did not qualify as human subjects research. Therefore IRB approval was not required.

\section{Study subjects (social media accounts)}

We used the search function of Twitter (TW) and Instagram (IG) on March 26, 2019, to generate a list of all accounts related to the following terms, each an independent text search: fertility, infertility, ttc (trying to conceive), egg freezing, ivf (in vitro fertilization), endometriosis, and reproductive. Many other search terms were considered (AMH, antimullerianhormone, PCOS, etc.), but the included terms were chosen based on (a) their likelihood to represent fertility/infertility as a whole and (b) the terms a new patient or novice user might search. Exclusion criteria included the following: (1) accounts not in English; (2) private accounts requiring approval for access to content; (3) accounts with no posts in more than 1 year; or (4) accounts with content unrelated to search terms.

\section{Variables and data collection}

Between March 31, 2019, and April 7, 2019, all included Twitter and Instagram accounts were assessed to determine author type, whether the author was a board-certified Reproductive Endocrinologist (REI-BC), the age of account, the number of followers, the number of posts, content of last 5 posts, and individual hashtags used in last 5 posts. An account identified as a potential board-certified REI (REI-BC) was further verified by confirmed licensure on the American Board of Obstetrics and Gynecology or ABOG website.

The 5 most recent posts were analyzed and classified, similar to previously published literature [8], by 3 independent reviewers (by majority or unanimity) into 1 of 10 categories: education/research (information related to medical facts or a published article), promotion (information about an event, product or service), inspiration/support (quotes, spiritual or community messages), personal story, celebrity story, humor, political (related to fertility, insurance or other political events), news (related to fertility, insurance or other news), outreach/awareness (public service announcements, etc.), or other. Given the high social media post volume and short cross-sectional timeframe for data collection, the number of posts (5) for review per account was chosen as a representative sampling.

Accounts were also assessed for influencer status. Concepts of user engagement, reach, and verification are 
important measures and were considered. However, these factors were ultimately excluded as they are not readily accessible to the general public. It is also important to note there is no standard or accepted definition for an influencer, even within a single social media platform. Therefore, based on marketing data and the concepts of influencer tiers [9] and that the fertility webspace would be smaller than the reach of a megainfluencer, such as a celebrity, influencer status was defined as greater than 10,000 followers per Instagram account [10] or a verified check mark on Twitter account, which is a blue badge placed by Twitter administration on accounts confirmed to be of public interest legitimizing their authenticity [11]. Authenticity and public interest are not in of themselves criteria for being an influencer, but given the account holder must apply for the blue badge and it must be confirmed, it was chosen for this study as the best marker in the current publicly available metrics.

\section{Analysis}

All social media accounts were categorized, and their characteristics were described as means with percentages or medians with ranges as appropriate. We compared the account characteristics between platforms, as well as between influencers and non-influencers, using independent $t$ tests. Finally, a classification and regression tree (CART) analysis was performed using account variables (activity and inclusion of most frequent content by social media platform) to determine factors associated with influencer status by social media platform. Misclassification of influencers (not identified as influencers by the algorithm) was weighed twice as high for priority in the model. The classification tree was confirmed by stepwise multiple logistic regression.

\section{Results}

Of 710 accounts identified, 347 were on Twitter and 363 were on Instagram. After excluding 192 accounts (69 TW, 104 IG), we included a total of 537 accounts in this study, 278 from

Table 1 Study sample inclusion and exclusion criteria

\begin{tabular}{llll}
\hline & \multicolumn{1}{l}{ Twitter } & \multicolumn{1}{l}{ Instagram } & \multicolumn{1}{l}{ Total } \\
\hline Accounts identified & \multicolumn{1}{l}{347} & \multicolumn{1}{l}{363} & \multicolumn{1}{l}{710} \\
Accounts included & $278(80.1 \%)$ & $259(71.3 \%)$ & $537(75.6 \%)$ \\
Accounts excluded: & $69(19.9 \%)$ & $104(28.7 \%)$ & $173(24.4 \%)$ \\
Not in English & $7(10.1 \%)$ & $5(4.8 \%)$ & $12(7.0 \%)$ \\
Private & $4(5.8 \%)$ & $59(56.7 \%)$ & $63(36.4 \%)$ \\
No posts in >1 year & $6(8.7 \%)$ & $16(15.4 \%)$ & $22(12.7 \%)$ \\
Unrelated content & $52(75.4 \%)$ & $24(23.1 \%)$ & $76(43.9 \%)$ \\
\hline
\end{tabular}

Twitter and 259 on Instagram (Table 1). Table 1 also shows the basis for account exclusion. The most common reason for exclusion of a Twitter account was unrelated content, for example; the search term "ttc" queried accounts related to entities such as "Train Transit Company" instead of content related to fertility, infertility, or trying to conceive. The most common exclusion indication for an Instagram account was for private accounts. A review of the included accounts revealed multiple types of account authors (Table 2), the most common being individual patients ( $n=162,30.2 \%$ accounts), support groups ( $n=123,22.9 \%$ accounts), infertility clinics ( $n=90$, $16.8 \%$ accounts), and accounts classified as "other" ( $n=75$, $14.0 \%$ accounts).

The average age of accounts was $84.2 \pm 11.5$ months for Twitter and $21.0 \pm 7.8$ months for Instagram. The median number of posts was 3542 "Tweets" (interquartile range [IQR] 1374.5-8960.5, full range 10-251,300) per Twitter account, and the median number of followers per account was 1317 (IQR 770.8-4005.3, full range 77-561,000). The median number of posts per Instagram account was 129.5 (IQR 53.3-334.0, full range 1-2784) with median number of followers per Instagram account 1008.0 (IQR 306.0-3345.3, full range 6-55,900).

There were 39 influencers identified on Twitter and 28 influencers identified on Instagram, comprising $12.5 \%$ of all accounts reviewed. Table 2 demonstrates the author types of influencers by social media platform. The average age of Twitter influencer accounts was $85.3 \pm 36.3$ months, and the average age of Instagram influencer accounts was $36.2 \pm$ 7.0 months. Influencer accounts on both platforms were older than non-influencer accounts (TW $102.3 \pm 26.5$ vs $84.2 \pm$ 11.5 months, $p<0.0017$; IG $39.1 \pm 7.0$ vs $21.0 \pm 7.8$, $p<0.0001$ ). The median number of posts ("Tweets") per Twitter influencer was 9113.5 (IQR 3850.0-17,750.0, full range 267-82,000.0), and median number of followers was 7062.5 (IQR 3065.0-32,550.0, full range 926-561,000.0). The median number of posts per Instagram influencer was 592.5 (IQR 298.8-1118.0, full range 97-2874) with the median number of follower per IG influencer 16,100.0 (IQR 13,650.0-263,000.0, full range 10,100-55,900). The most represented author types of influencer accounts were awareness/advocacy/support (59.8\% TW, $25.0 \%$ IG), individual patients (12.8\% TW, 25.0\% IG), or accounts classified as "other" (17.9\% TW, 21.0\% IG). Most importantly, only $7.7 \%(n=3)$ of Twitter and $7.1 \%(n=2)$ of Instagram influencers, a total of 5 influencers on both platforms, were board-certified infertility specialists.

The 5 most recent posts on each account were analyzed for content (Fig. 1) and categorized as described. A total of 1390 posts were reviewed from the included Twitter accounts of which the most frequent content was promotion $(28.2 \%)$ and research or education $(20.2 \%)$. A total of 1290 posts were reviewed from the included Instagram accounts with the most 
Table 2 Breakdown of account author types and identification of board-certified reproductive endocrinologists and influencers

\begin{tabular}{|c|c|c|c|c|c|c|}
\hline \multirow[t]{2}{*}{ Account author type (listed by frequency) } & \multicolumn{3}{|l|}{ Instagram } & \multicolumn{3}{|l|}{ Twitter } \\
\hline & $\begin{array}{l}\text { Total } \\
\text { number } \\
\text { accounts }\end{array}$ & $\begin{array}{l}\text { Number of board } \\
\text { certified REI }\end{array}$ & $\begin{array}{l}\text { Number of } \\
\text { influencers }\end{array}$ & $\begin{array}{l}\text { Total } \\
\text { number } \\
\text { accounts }\end{array}$ & $\begin{array}{l}\text { Number of board } \\
\text { certified REI }\end{array}$ & $\begin{array}{l}\text { Number of } \\
\text { influencers }\end{array}$ \\
\hline Patients & 95 & 0 & 7 & 67 & 0 & 4 \\
\hline Awareness, advocacy, and support accounts & 48 & 0 & 7 & 75 & 0 & 23 \\
\hline Infertility clinic & 48 & 48 & 0 & 42 & 40 & 1 \\
\hline Other & 32 & 0 & 6 & 43 & 0 & 7 \\
\hline $\begin{array}{l}\text { Other allied health professionals (nurses, } \\
\text { embryologists, midwives, non-REI doctors) }\end{array}$ & 10 & 0 & 2 & 18 & 0 & 1 \\
\hline REI MD/DO & 9 & 9 & 1 & 15 & 11 & 1 \\
\hline $\begin{array}{l}\text { Wellness space accounts - naturopaths, holistic fertility } \\
\text { "experts," acupuncturists, fertility coaches }\end{array}$ & 14 & 0 & 4 & 9 & 0 & 0 \\
\hline Organizations with MD advisory boards & 3 & 2 & 1 & 5 & 2 & 1 \\
\hline Academic or professional society & 0 & 0 & 0 & 4 & 1 & 1 \\
\hline Total & 259 & 59 & 28 & 278 & 54 & 39 \\
\hline
\end{tabular}

frequent content being personal stories (31.7\%) and inspiration or support $(23.7 \%)$. A review of the individual hashtags used per post on both social media platforms was performed with the top hashtags by use shown in Fig. 2. A total of 1581 hashtags were reviewed, 575 on Twitter and 1006 on Instagram. Hashtag analysis revealed that certain hashtags, despite being similar in nature had discrepant use, for example \#infertility (128 uses) compared with \#infertile (2 uses) and \#ttc (54 uses) compared with \#tryingtoconceive (5 uses).

Finally, a classification and regression tree (CART) analysis was performed to create a prediction model to identify influencer accounts and determine factors that best predicted classification as an influencer by social media platform. The model utilized high or low activity (defined as $>50$ posts/ month on Twitter and $>10$ posts/month on Instagram) as well as inclusion of the most frequent content in posts per platform (promotion, inspiration, education on Twitter; personal story, inspiration or promotion on Instagram). Figure 3 depicts the nodal tree from the CART analysis; on both platforms an account with high activity was the best predictor for classification as an influencer. Promotional content on Twitter and inspirational content on Instagram were the next best classifiers but were not the most predictive of classification. Logistic regression utilizing the same variables confirmed that only high activity per platform was significant for being an influencer (Twitter B $-1.25, p<0.002$; Instagram B -1.32 , $p<0.004)$.

\section{Discussion}

To our knowledge, this is the first study to describe the topography of the fertility-related social media landscape as well as the first to highlight the paucity of board-certified REI physicians in this webspace. We found that the majority of accounts in the fertility-related social media landscape are patients, inspirational accounts, and/or support groups, and that these accounts were more influential than the physician, academic society, and fertility clinic accounts. Additionally, we found that the most frequent content were individual stories and inspirational posts. However, even armed with the most popular content, the majority of fertility specialists did not meet criteria for being a social media influencer. Overall, Twitter accounts did have a higher proportion of posts providing educational facts and even research articles. However, the authors of these posts were most often individual patients and less likely to be physicians. In turn, while a patient may share an interesting study article or new treatment, the interpretation of the study's findings or the new treatment's appropriate usage may be incorrectly communicated. Together, these findings provide an important message to all those in the fertility community. First, this serves as a reminder to consider the type and source of information that patients may come to us with and how this knowledge was acquired. Second, physicians should be aware of what is being discussed within the fertility webspace to engage with our patients on these topics to ultimately strengthen physicianpatient relationships. Finally, we hope these findings engage all board-certified or eligible REI physicians providing information in this space to ensure reliable and correct messaging regarding fertility care, research, and treatment to empower patients to make self-informed decisions.

The topic of social media and influence in medicine has been discussed in other domains of healthcare. A review of the literature shows many fields, ranging from epilepsy, cardiovascular medicine, and plastic surgery as well as pathology [12-15], have investigated this area and come to varying 
Fig. 1 Content analysis by social media platform

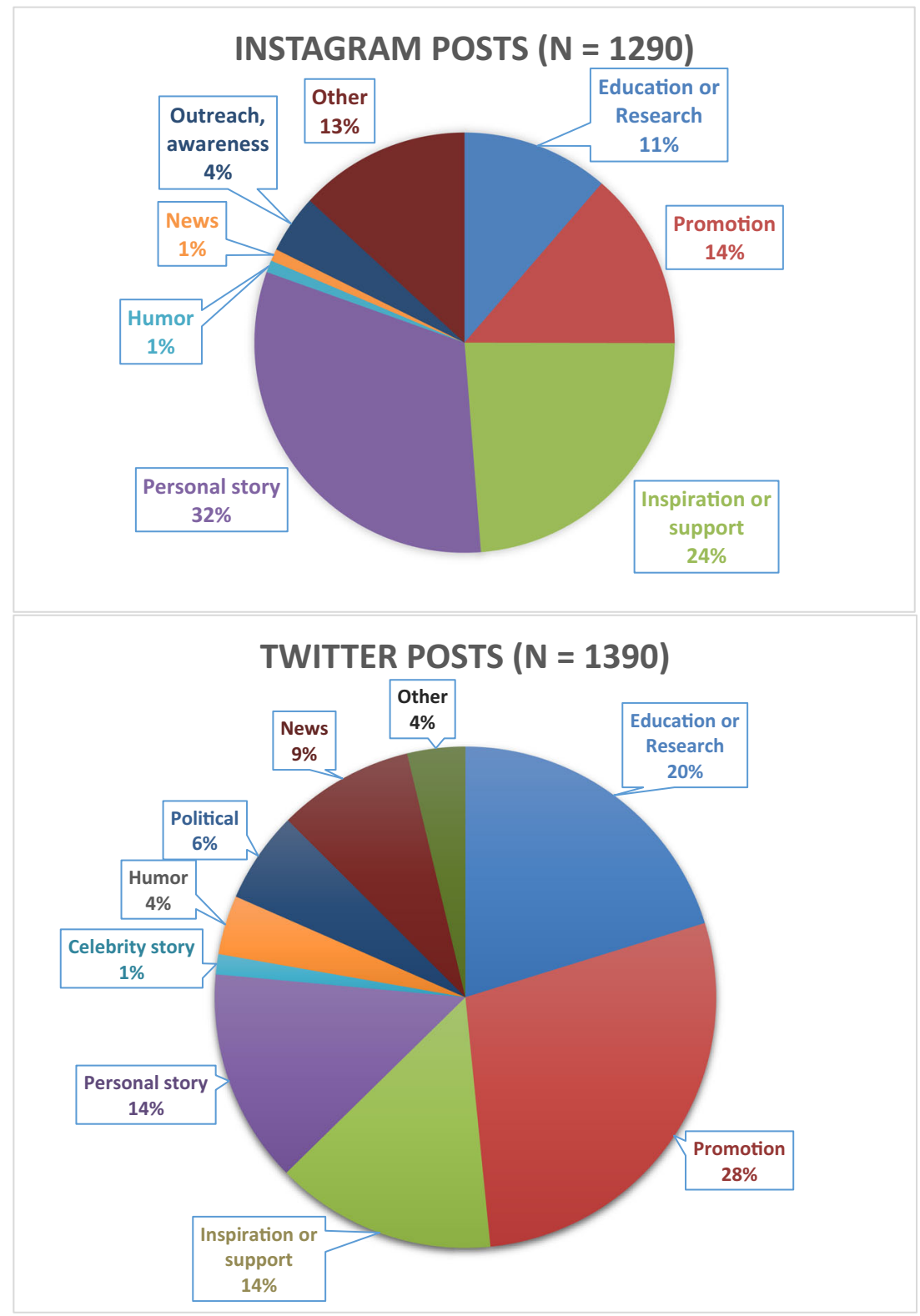

conclusions on efficacy and implications. A review of published literature on social media in healthcare by Gupta et al. found 139 articles and abstracts and reviewed 26 of these publications from Plastic Surgery publications [15]. The authors concluded that the while social media can be beneficial, the role of an influencer in medicine must be delicately balanced within the ethics of medicine, and this balance has not yet been fully addressed in terms of best practices [15]. An analysis of the use of social media platforms (Twitter, Facebook, Instagram, LinkedIn, and Doximity) and their associated use within academic Pathology programs showed high utilization with opportunities for networking and employment but had similar cautions of potential patient privacy issues. For example, when sharing relevant stories or images, one must ensure informed consent has been obtained [14].
However, other fields, for example Cardiovascular Medicine, continue to highlight the potential and perspective social media can offer, with worldwide instantaneous communication to perhaps educate patients prior to their office visits, and present a vision for best practice and the future of medicine as it grows with social media [13]. While the platforms and landscape may change, digital connectivity and social media is likely here to stay. Physicians may be able to benefit from practice in this space to improve communication with patients and dispel inaccurate information dispersed by others within social media. In particular, given the perpetual "youth" of those presenting to fertility clinics, and that these cohorts are often avid consumers of new technology, staying connected and competent within social media will likely be paramount for the modern fertility physician. 
Fig. 2 Top 11 individual hashtags by frequency on social media

\section{Individual hashtag uses $(\mathrm{n}=605)$}

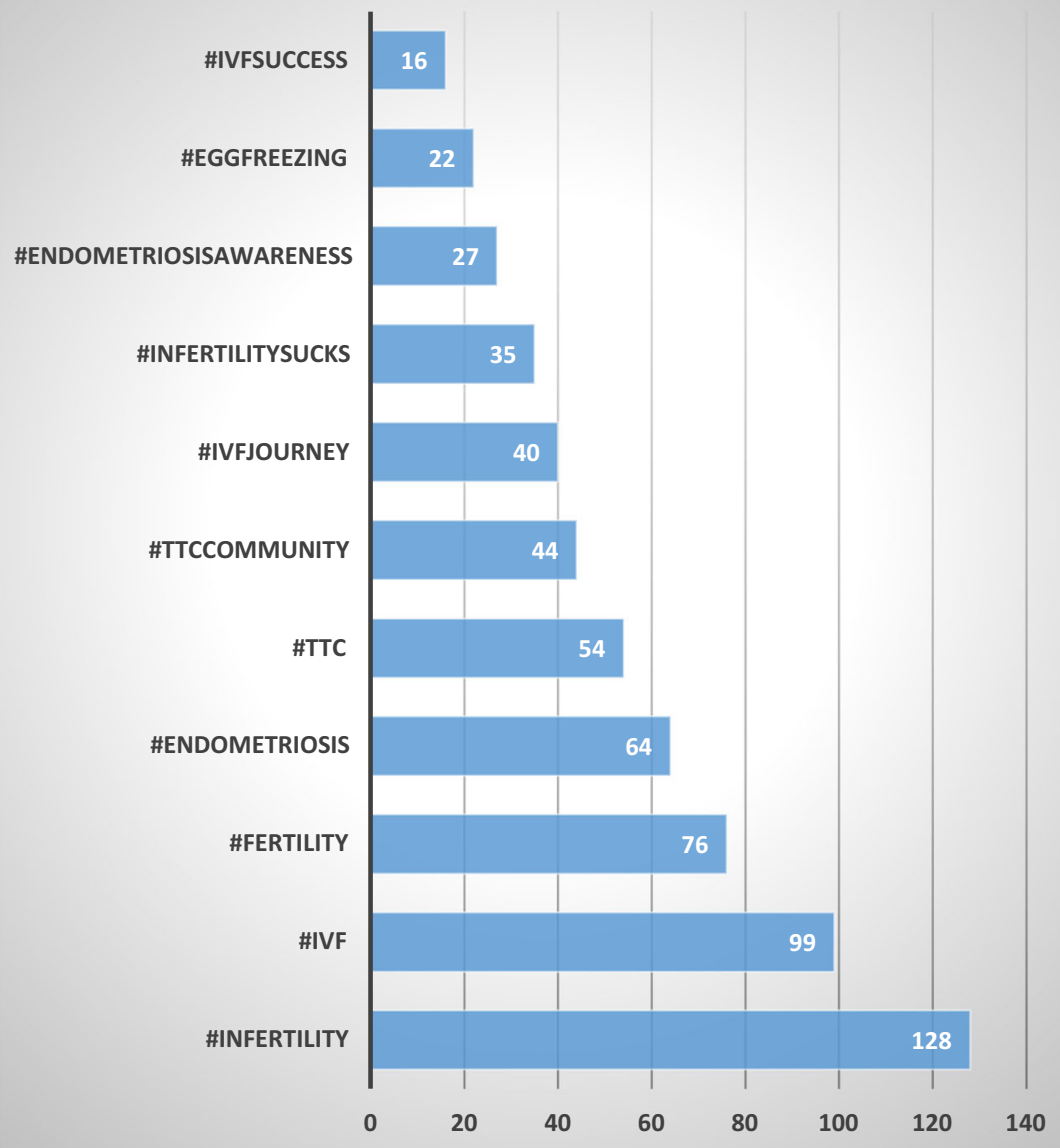

Our study has three important implications. First, it shows that fertility and infertility are a highly active area of social media. In our opinion, this activity level will continue to evolve and expand and, therefore, it may behoove REI physicians not only to participate in this space but also to establish themselves as effective and trustworthy communicators with their patients who are, most likely, already utilizing social media. Second, armed with this information, we believe that REI physicians can be proactive and creative to gain more influential status. Many of the accounts included in this study were extremely active and collaborative with their platforms, but perhaps there are better, more effectual ways we can guide our efforts to obtain wider or more "influential" platforms. Finally, we believe that our results show that REI physicians can be "smarter" about managing their posts. One of the great aspects of social media is that each platform allows people to frame their own experiences however they so choose. In fact, the recently launched professional society, Association for Healthcare Social Media or AHSM, was created specifically for the use of social media by health care professionals with the goal of "positively influencing public health and health care through social media" [16]. If physicians, REI included, could combine education with patient stories or inspirational content on a more frequent basis, our collective voice as healthcare influencers could be much more powerful and possibly, as suggested, provide a boost for dissemination of publication and academic promotion [17].

As mentioned, the principal strength of our study is that it is the first, largest, and most representative evaluation of the fertility webspace as well as of the characteristics of a fertility influencer. However, our study has several limitations. Due to the nature of the study design, our data only represents a static moment in time and therefore does not characterize the dynamics of social media. Additionally, our study does not consider the evolution of an individual account over time or which accounts may be "becoming" influential. This temporal bias is further highlighted with the restriction to 5 posts reviewed per account. We acknowledge that inclusion of all posts for each accounts would be a better representation of each account; however, this was simply not feasible in the design of the study. The nature of social media and its fastpaced and constantly changing nature make capturing these variables difficult. 


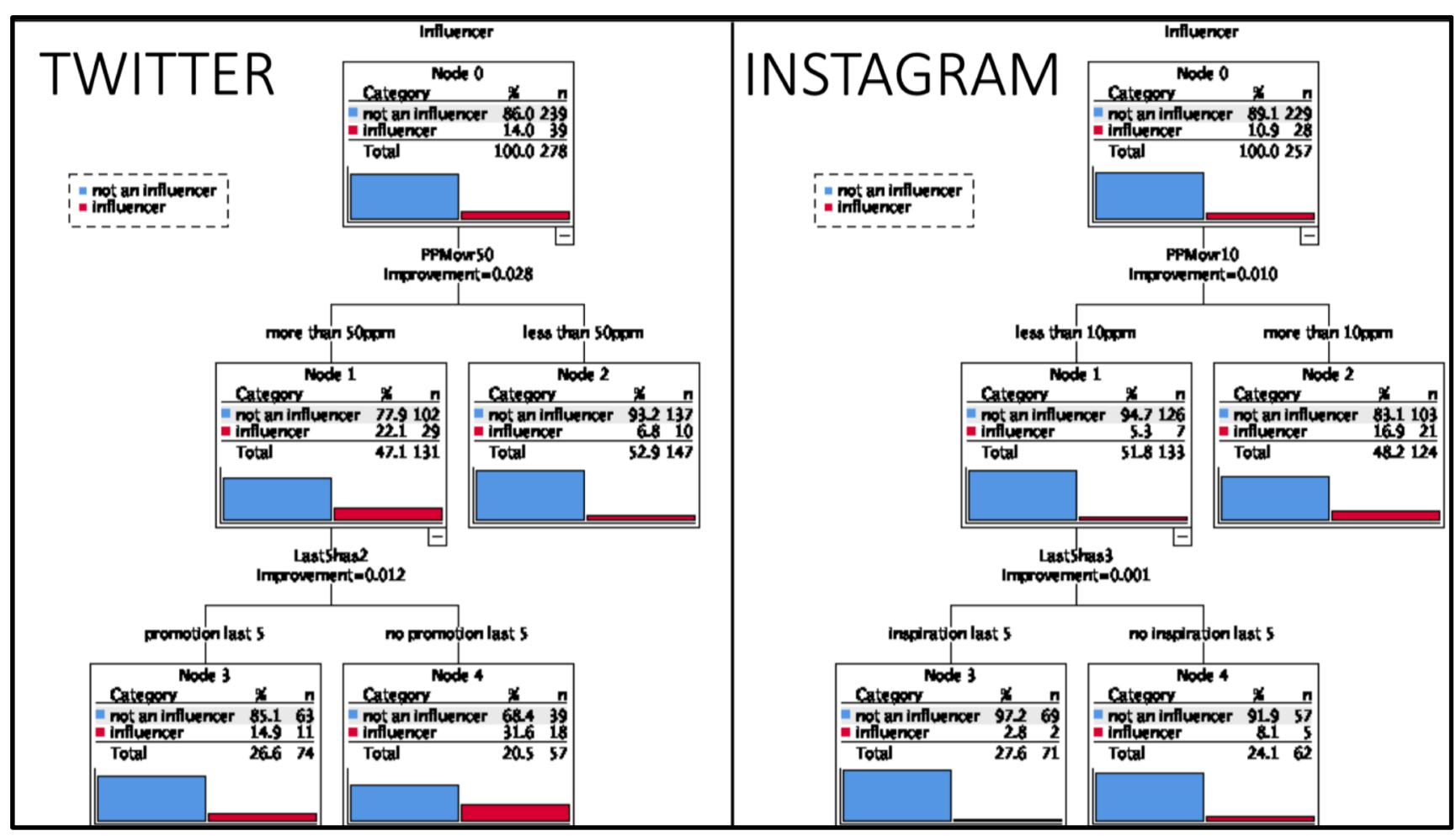

Fig. 3 Cart analysis flowcharts

Another considerable limitation is that our study did not include all accounts related to infertility and fertility. First, private social media accounts were excluded. It is possible that the content and "influential" factors from these private accounts differ significantly from those with public access. However, we hypothesize that these accounts, while likely extremely active, have a similar post content and frequency. Second, the search function for Instagram only queries the handle and the first line of the user biography. Therefore, many users who had bios with included search terms but not in the "searchable" area were also excluded. This is an important limitation. However, we argue that this is how a novice user on the platform would be using the interface and may be the best representation of the most accessed accounts. It could also be an important point to all future social media users on how to be "best searched." The methodology for verifying REI-BC certification was not able to verify individuals that are REI board eligible such as REI Fellows or recent graduates prior to the certifying exam. However, no such individuals were identified by the search methodology for this study, and thus, this limitation was not encountered. Nonetheless, we recognize the limitation remain that the content and influencer information from these private and "unsearched" accounts were not included in our study. Finally, we recognize that there are metrics other than the number of followers that play into social media influence, such as reach and engagement. However, these metrics are not publicly available, whereas follower number is. Thus, our methodology was restricted by the nature of publicly available metrics, yet still represents the organic user experience.

There are many questions that remain unanswered that warrant future research considerations. Do influencers change behavior of their followers? Has social media outreach and exposure increased awareness about fertility issues and, more importantly, is it changing access to care? Moreover, an analysis of the factors involved in building an influential account and, in turn, that account's network is also crucial. Who is following whom? Does being followed by certain accounts (e.g., other influencers) gain you more credibility, followers, and/or influence in a certain webspace? What can we learn from interactions such as re-tweets or re-posts? Perhaps, taking notes from successful influencers in other spaces can help physicians active in social media best utilize their time in building an influential, impactful account.

The answers to these questions will continue to be explored, answered, and improved. While metrics such as frequent activity and a large following or verification were discussed in this study, they are not the definitive endpoints and nor should they be viewed as barriers to begin or continue to engage with social media. At the time of submission of this manuscript, the impact of the COVID-19 pandemic was just beginning and with it physicians embarking on novel ways to engage their patients, such as with virtual healthcare platforms. Mechanisms, metrics, and perhaps needs will continue 
to evolve but at least one point appears to be clear nowsocial media is here to stay and will likely to expand. Therefore, we encourage those with interest in this webspace to get involved. A positive influence-for a patient, for a field, or for healthcare as a whole - may start with a single post.

In conclusion, the power of social media has been realized in several industries, but has yet to be fully harnessed in healthcare. Our study shows that the infertility social media webspace is active and fertility influencers exist. We believe that REI physicians may be the best suited and capable to do more to engage, educate, and influence in this domain to better communicate and inform our patients.

Acknowledgments The authors gratefully acknowledge the account creators on the social media platforms.

Author contributions All authors contributed to the writing of the manuscript. All authors read and approved the final manuscript.

Compliance with ethical standards All procedures performed in this study were in accordance with the ethical standards of the institution. There were no human subjects as part of this manuscript and therefore no need for informed consent.

Conflict of interest The authors declare that they have no conflict of interest.

\section{References}

1. Hackworth BA, Kunz MB. Healthcare and social media: building relationships via social networks. Acad Health Care Manag J. 2011;7(2):1-14.

2. Kane G, Fichman RG, Gallaugher J, Glaser J. Community relations 2.0. Harv Bus Rev. 2009;87(11):45-50 132.

3. Pew Research Center. Gender divide on social media use tilts toward men in developing economies, women in advanced economies. https://www.pewresearch.org/global/2018/06/19/3-socialnetwork-adoption-varies-widely-by-country/pg_2018-06-19_ global-tech 3-03/ Accessed August 20 ${ }^{\text {th }}, 2019$.

4. Broughton $\bar{D}$, Schelble A, Cipolla K, Cho M, Franasiak J, Omurtag KR. Social media in the REI clinic: what do patients want? J Assist Reprod Genet. 2018;35(7):1259-63.
5. Hammarberg K, Zosel R, Comoy C, Robertson S, Holden C, Deeks $\mathrm{M}$, et al. Fertility-related knowledge and information-seeking behaviour among people of reproductive age: a qualitative study. Hum Fertil (Camb). 2017;20(2):88-95.

6. @ katieaka. New research: the value of influencers on Twitter https://blog.twitter.com/marketing/en_us/a/2016/new-research-thevalue-of-influencers-on-twitter.html. Accessed August $20^{\text {th }}, 2019$.

7. Perrin A, Anderson M. Share of U.S. adults using social media, including Facebook, is mostly unchanged since 2018. Pew Research Center. https://www.pewresearch.org/fact-tank/2019/04/ 10/share-of-u-s-adults-using-social-media-including-facebook-ismostly-unchanged-since-2018/. Accessed April 10 ${ }^{\text {th }}, 2019$.

8. Omurtag K, Jimenez PT, Ratts V, Odem R, Cooper AR. The ART of social networking: how SART member clinics are connection with patients online. Fertil Steril. 2012;97(1):88-94.

9. Influencer tiers for the influencer marketing industry. https:// mediakix.com/influencer-marketing-resources/influencer-tiers/. Accessed April 10 2019.

10. Lieber C. How and why do influencers make so much money? The head of an influencer ageny explains. https://www.vox.com/thegoods/2018/11/28/18116875/influencer-marketing-social-mediaengagement-instagram-youtube. Accessed April $7^{\text {th }}, 2019$.

11. Twitter Help Center. https://help.twitter.com/en/managing-youraccount/about-twitter-verified-accounts. Accessed September $21^{\mathrm{st}}$, 2019.

12. Sultan M, Brown EM, Thomas RH. Clinicians embracing social media: potential and pitfalls. Epilepsy Behav. 2019;12:1064262.

13. Parwani P, Choi AD, Lopez-Mattei J, Raza S, Chen T, Narang A, et al. Understanding social media: opportunities for cardiovascular medicine. J Am Coll Cardiol. 2019;73(9):1089-93.

14. Isom J, Walsh M, Gardner JM. Social media and pathology: where are we now and why does it matter? Adv Anat Pathol. 2017;24: 294-303.

15. Gupta N, Dorfman R, Saadat S, Roostaeian J. The plastic surgery social media influencer: ethical considerations and a literature review. Aesthet Surg J. 2019; In press. https://doi.org/10.1093/asj/ sjz329.

16. Association for Healthcare Social Media. https://ahsm.org. Accessed December $9^{\text {th }}, 219$.

17. Lee G, Choi AD, Michos ED. Social Media as a means to disseminate and advocate cardiovascular research: why, how, and best practices. Curr Cardiol Rev. 2019; In press. https://doi.org/10. 2174/1573403X15666191113151325.

Publisher's note Springer Nature remains neutral with regard to jurisdictional claims in published maps and institutional affiliations. 